\title{
Tuberculosis ocular
}

\section{Elena De Benedetti Z., Bárbara Carranza L., Eduardo Gotuzzo H. e Isaías Rolando C.}

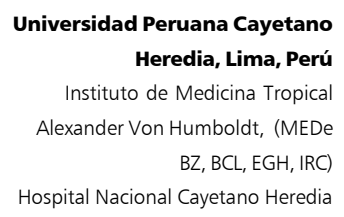

$(\mathrm{EGH}, \mathrm{IRC})$

Recibido: 13 de noviembre de 2006 Aceptado: 10 de abril de 2007

Correspondencia a: Eduardo Gotuzzo Herencia egotuzzo@gotuzzos.com

\section{Ocular tuberculosis}

Tuberculosis (TB) is considered a public health problem in several countries. This disease is classified as either pulmonary or extrapulmonary. Within the extrapulmonary disease, ocular involvement is uncommon but it is important to recognize it because its incidence has been reported up to $1 \%$. Ocular TB cases can be divided in primary and secondary. These manifestations can be caused by an active infection that invades the eye or by an immunologic reaction of delayed hypersensitivity in absence of the infectious agent. The most common clinical presentations are: chronic anterior uveitis, choroiditis and sclerokeratitis. Despite the existence of highly sensitive molecular diagnostic techniques, the diagnosis of ocular tuberculosis continues to be presumptive, based upon clinical presentation, systemic evaluation and response to treatment. For the treatment we use four drugs during a two month period (isoniazid, rifampin, pyrazinamide and ethambutol) and two drugs for four additional months.

Key words: ocular tuberculosis, ocular manifestations, choroidal tubercles, uveitis, diagnosis.

Palabras claves: Tuberculosis ocular, manifestaciones oculares, tubérculos coroideos, uveítis, diagnóstico.

\section{Introducción}

L a infección por Mycobacterium tuberculosis es la segunda causa de mortalidad por un agente infeccioso en el mundo, luego del virus de inmunodeficiencia humana (VIH), determinando 1,8 millones de muertes por año. Noventa y ocho por ciento de estas muertes ocurren en países en vías de desarrollo como Perú ${ }^{1}$. En los últimos años, la tuberculosis (TBC) ha sido considerada un problema de salud pública reemergente ${ }^{2,3}$.

Datos del Ministerio de Salud de Perú indican que la incidencia de $\mathrm{TBC}$ ha ido en disminución en los últimos años. En un reporte hecho por la Organización Mundial de la Salud (OMS) en el año 2004, la incidencia de TBC en Perú fue de 178 casos por 100.000 habitantes/año. Según este reporte, la tendencia de la misma era a disminuir en $5,7 \%$ cada año ${ }^{4}$.

En el mundo, la incidencia de TBC varía considerablemente; en Latinoamérica encontramos países con baja incidencia como Cuba y Chile (7,5-14 casos por 100.000 habitantes, respectivamente) y países con una incidencia elevada como Perú y Haití (115-169 casos por 100.000 habitantes, respectivamente). Existen 23 países considerados "hot countries" por la alta incidencia de TBC. Estos países concentran el $80 \%$ de todos los casos de TBC en el mundo. Desde el año 2003, Perú ya no forma parte de esta lista debido a los resultados obtenidos con el cumplimiento de las metas establecidas por el Programa Nacional de Control de la Tuberculosis (PCT) y reconocidos por la OMS.

\section{Epidemiología}

Actualmente, en el mundo existen 1.900 millones de personas infectadas por $M$. tuberculosis, de las cuales $10 \%$ desarrolla la enfermedad. Cada año hay 8,2 millones de nuevos casos diagnosticados ${ }^{1}$. Tuberculosis es la causa de $7 \%$ de todas las muertes y continúa siendo la enfermedad a tratar con mayor impacto costo-efectivo ${ }^{5}$.

Los casos de TBC ocular han sido documentados desde el siglo $\mathrm{XIX}^{6,7}$. Su descripción más antigua se atribuye a Maitre-Jan quien, en el año 1711 describió el caso de un paciente con una lesión en el iris, de la cual pensó se trataba de TBC. En 1830, Gueneau de Mussy reconoció la presencia de tubérculos coroideos en TBC miliar, y en 1855 Jaeger hizo la primera descripción anatómica de los mismos. A pesar de ello, no fue sino hasta 1882 que el bacilo causal de la TBC fuera descubierto por Robert Koch y un año después, en 1883 von Michell identificó plenamente el microorganismo en el ojo humano por primera vez y consideró a la TBC como causa común de uveítis ${ }^{8}$.

La TBC ocular es una entidad poco frecuente y su impacto no ha sido bien reconocido. En algunas se- 
ries, su incidencia puede llegar a $1 \% \%^{2,9,10}$. Puede comprometer cualquier componente del sistema visual, desde los párpados hasta el nervio óptico. Las presentaciones más comunes son: uveítis anterior crónica, coroiditis y esclero-queratitis ${ }^{2}$. La mayoría de los pacientes con TBC ocular no tienen historia de enfermedad pulmonar o sistémica y hasta $50 \%$ tiene una radiografía de tórax normal ${ }^{11}$.

En los pacientes con TBC ocular no se ha encontrado diferencia en la incidencia entre grupos etarios ${ }^{12}$; sin embargo, se describe que la TBC extra-ocular es más frecuente en niños. La TBC orbitaria puede ocurrir a cualquier edad, pero es más frecuente en las primeras dos décadas de la vida; por ejemplo, los tubérculos coroideos son más frecuentes en lactantes, niños y adultos jóvenes. La pan-oftalmitis es más común en niños o adultos gravemente enfermos. La TBC crónica diseminada de la coroides, se observa con más frecuencia en la segunda y tercera década, siendo rara después de los 50 años.

En la literatura científica no se ha reportado si el género es un factor a considerar para el desarrollo de TBC ocular. En una serie de 200 pacientes con diagnóstico de uveítis, se encontró que $21(10,5 \%)$ tenían TBC. En esta serie, se encontró que la relación hombre: mujer fue de 3:2 (hombres 15 casos $(71,4 \%$ ), mujeres 6 casos $(28,6 \%)$ con un $\mathrm{p}<0,05)^{12}$.

Se clasifica a la TBC en pulmonar y extra-pulmonar, siendo más frecuente y mejor estudiada la forma pulmonar. Antes de la era del síndrome de inmunodeficiencia adquirida (SIDA), 83\% de los casos de TBC estaban limitados al pulmón y el $17 \%$ restante eran casos extra pulmonares ${ }^{2}$. Actualmente, la TBC extra-pulmonar es más frecuente y representa 40 a $50 \%$ de los casos. La prevalencia de TBC en pacientes con SIDA reportada por la Organización Panamericana de la Salud es de 63,8\% para Haití, 34,2\% para Perú, 24,8\% para Panamá, 13,7\% para Guyana, 11,5\% para El Salvador $9 \%$ para Honduras y $2,5 \%$ para Nicaragua ${ }^{1}$. Sin embargo, no hay muchas publicaciones sobre TBC ocular en pacientes con SIDA.

\section{Formas clínicas y tipos de lesión}

El compromiso ocular es un evento poco común en TBC. Las manifestaciones oculares asociadas a esta enfermedad pueden ser causadas por una infección activa que invade el ojo (en la cual se encuentra presencia del microorganismo en los tejidos oculares) o por una reacción inmunológica de hipersensibilidad retardada tipo IV a diversos componentes antigénicos de la propia micobacteria, en ausencia del agente infeccioso, como se observa en la patogenia del eritema nodoso o eritema indurado de Bazin, y que sería la responsable de diversas lesiones oculares como la vasculitis retiniana $^{8,13}$. En el futuro, mientras se aprenda más de la respuesta inmune a antígenos externos, podremos encontrar que muchas condiciones que empiezan como infecciones o que puedan ser exacerbadas por infecciones secundarias, pueden más tardíamente ser perpetuadas por mecanismos inmunológicos no regulados ${ }^{14}$.

Las manifestaciones clínicas de la TBC ocular varían dependiendo de la virulencia de la micobacteria, de la resistencia del huésped a la misma y del grado de hipersensibilidad tisular a ella ${ }^{8}$.

Los casos de TBC ocular se pueden dividir en primarios y secundarios ${ }^{15}$. En la TBC ocular primaria, el ojo ha sido la puerta de entrada de la micobacteria y no existe evidencia de infección sistémica. Esta forma de infección está limitada a enfermedad conjuntival y corneal. Es extremadamente inusual que una infección ocular primaria evolucione hacia un cuadro de TBC sistémica, aunque su ocurrencia ha sido previamente sugerida $^{6}$. El foco primario es generalmente un proceso ya curado (que puede no haber dejado secuela), o corresponde a formas sub-clínicas, y justamente la lesión ocular lo evidencia. Es así que Woods sólo encontró el foco inicial de TBC en $40 \%$ de los enfermos con TBC ocular comprobada ${ }^{16}$.

La forma secundaria se define como una infección que resulta de la diseminación contigua, a partir de una estructura adyacente, o por diseminación hematógena, por ejemplo a partir del pulmón, siendo ésta la más común ${ }^{17}$. Sin embargo, las manifestaciones intraoculares de TBC están, generalmente, asociadas a infección sistémica y son, por lo tanto, consideradas como TBC secundaria o extra-pulmonar ${ }^{18}$.

Las manifestaciones más comunes son: iridociclitis granulomatosa crónica, tuberculomas coroidales o periflebitis retinal, siendo los tuberculomas coroideos la forma mejor documentada y un indicativo de diseminación hematógena ${ }^{19,20}$. En la Tabla 1 se describen las formas clínicas descritas en tres series reportadas por diferentes autores.

La TBC ocular puede presentarse como una malignidad ocular $^{23}$. En una serie de cinco pacientes referidos a un centro oftalmológico para descartar la presencia de tumores oculares, se encontró que un paciente tenía pan-oftalmitis y otro, endoftalmitis que terminó en perforación escleral. Dos pacientes tuvieron un granuloma coroidal activo asociado a uveítis y un paciente tuvo lesión coroidal amelanótica sin signos inflamatorios. De estos cinco casos, dos tenían una historia conocida de TBC sistémica, mientras que los hallazgos oculares en los otros tres casos fueron la manifestación inicial del cuadro de TBC sistémica ${ }^{2}$. 
Tabla 1. Formas clínicas de presentación de tuberculosis ocular

\begin{tabular}{|c|c|c|c|}
\hline & $\begin{array}{l}\text { Demirci et al } \\
\text { Año 2004*2 }\end{array}$ & $\begin{array}{l}\text { Donahue HC. } \\
\text { Año } 1967^{21}\end{array}$ & $\begin{array}{c}\text { Morimura } Y \text { et al } \\
\text { Año } 2002^{22}\end{array}$ \\
\hline$N^{\circ}$ de casos de TBC ocular & 92 & 154 & 10 \\
\hline \multicolumn{4}{|l|}{ Manifestación clínica } \\
\hline Masa coroidal (\%) & 34 & - & 20 \\
\hline Coroiditis/corio-retinitis (\%) & 27 & 30 & 40 \\
\hline Vitreitis (\%) & 24 & - & - \\
\hline Uveítis anterior (\%) & 13 & - & - \\
\hline Pan-oftalmitis (\%) & 11 & - & - \\
\hline Otros (\%) & - & $70 * *$ & $40 * * *$ \\
\hline \multicolumn{4}{|c|}{$\begin{array}{l}\text { *Los pacientes tenían más de una lesión. }{ }^{* *} \mid \text { ritis } 18,2 \% \text {; esclero-queratitis } 15 \% \text {; queratitis } \\
\text { intersticial 9,1\%; escleritis } 9,1 \% \text {; otros diagnósticos: } 18,6 \% \text {. }{ }^{* * *} 10 \% \text { nódulo del disco ópti- } \\
\text { co, } 30 \% \text { vasculitis retinal }\end{array}$} \\
\hline
\end{tabular}

Asimismo, hay reportes en la literatura en los cuales la endoftalmitis tuberculosa con leucocoria y una masa en el vítreo asociada a calcificación focal, simuló un retinoblastoma. La TBC es bien conocida por conducir a presentaciones ocultas y muy variadas. El caso anteriormente mencionado trae a nuestra atención el hecho que la TBC ocular puede presentarse también con calcificación intraocular distrófica ${ }^{24}$.

Singh et al, revisaron retrospectivamente 1.233 historias clínicas de pacientes con uveítis durante el período 1997-2001. El diagnóstico de TBC ocular fue hecho cuando el paciente presentaba sospecha clínica de enfermedad (uveítis granulomatosa anterior, periflebitis activa, neuro-retinitis, retino-coroiditis o absceso/granuloma) más uno de los siguientes: evidencia de enfermedad (punción de humor acuoso o vítreo positivo por reacción de polimerasa en cadena (RPC), baciloscopia positiva o cultivo positivo, o ambos; evidencia histopatológica de TBC en la biopsia de un ganglio linfático cervical o parahiliar y cualquiera de las dos siguientes: PPD $\geq 20 \mathrm{~mm}$, necrosis o radiografía de tórax anormal), y repuesta al tratamiento antituberculoso. En este estudio, se pudo encontrar una etiología específica en 602 pacientes $(48,2 \%)$. De éstos, $179(29,7 \%)$ fueron de etiología infecciosa, de los cuales TBC fue la infección más común en 125 pacientes $(69,8 \%)^{25}$.

En un estudio prospectivo, se evaluó el compromiso ocular en pacientes con TBC. Se seleccionó uno de cada dos pacientes con evidencia de TBC (baciloscopia positiva o cultivo positivo) hasta que se encontraron 100 casos. Todos los pacientes fueron sometidos a un examen oftalmológico. Se usaron los siguientes criterios para diagnosticar $\mathrm{TBC}$ ocular:
- Comprobada: Pacientes en los que se aisló $M$. tuberculosis de cualquier muestra ocular.

- Probable: Pacientes con M. tuberculosis en alguna muestra extra-ocular, lesiones oculares compatibles con TBC y no atribuibles a otras causas y una adecuada respuesta de las lesiones oculares a la terapia antituberculosa.

- Posible: Los mismos criterios que para la anterior, pero que la evolución clínica no se pudo evaluar.

Veintisiete de los pacientes tuvieron hallazgos oculares anormales (Tabla 2), nueve de ellos no relacionados a TBC, mientras que 18 tuvieron TBC ocular. Cumplieron con los criterios de probabilidad diez pacientes y ocho con los de posibilidad. Ningún caso cumplió con los criterios de TBC ocular comprobada. De los 18 que tuvieron TBC ocular, siete tuvieron compromiso ocular sintomático, de los cuales cinco presentaron pérdida de la visión. En 17 de los pacientes se encontraron lesiones coroidales, las cuales fueron coroiditis activa (nódulos o tuberculomas coroideos) o una cicatriz coroidal. La alta incidencia de compromiso coroidal se puede explicar por la gran irrigación sanguínea de esta estructura en el ojo. La Tabla 2 muestra las manifestaciones oculares de TBC en los 18 pacientes $^{26}$.

En este estudio, además, se encontró que la TBC miliar se asoció con más frecuencia a lesión ocular en general.

Tabla 2. Manifestaciones oculares de tuberculosis en 18 pacientes del Hospital General Universitario "Gregorio Marañón" , Madrid - España

\begin{tabular}{|c|c|}
\hline $\begin{array}{l}\text { Lesiones por TBC } \\
n=18 \text { pacientes }\end{array}$ & $\begin{array}{l}\text { Número de lesiones } \\
\text { oculares } \\
n=36^{*}\end{array}$ \\
\hline Nódulos coroideos & 12 \\
\hline Cicatriz coroidal & 8 \\
\hline Papilitis & 3 \\
\hline Lesión macular & 3 \\
\hline Vasculitis & 2 \\
\hline Vitritis & 2 \\
\hline Tuberculosis coroidea & 1 \\
\hline Dacrio-adenitis & 1 \\
\hline Escleritis posterior & 1 \\
\hline Precipitados periqueráticos & 1 \\
\hline Uveítis anterior & 1 \\
\hline Desprendimiento de retina & 1 \\
\hline
\end{tabular}




\section{Enfermedad externa}

\section{Párpados}

La TBC palpebral es más común en niños. Puede presentarse como un absceso frío (una masa fluctuante blanda sin inflamación aguda) o puede simular un chalazion $^{10}$.

\section{Conjuntiva}

La infección primaria es poco usual y afecta mayormente a niños. Generalmente, se trata de una enfermedad crónica que puede llevar a cicatrización del tejido involucrado. Los pacientes se presentan con conjuntivitis crónica unilateral, acompañada de una masa conjuntival o ulceración y asociada a linfo-adenopatía regional $^{6}$. En el examen puede evidenciarse descarga mucopurulenta y edema palpebral asociado a linfoadenitis regional tal como el síndrome óculo-glandular de Parinaud, el cual no se encuentra presente en la gran mayoría de las conjuntivitis bacterianas y alérgicas y es menos común en la conjuntivitis viral. Esta linfoadenitis es importante, ya que contribuye al diagnóstico diferencial puesto que, en otras formas de conjuntivitis (bacteriana o alérgica), no se encuentra ${ }^{10}$.

Los casos de TBC conjuntival han sido clasificados clínicamente como ulcerativa, tubérculo-miliar, granulación hipertrófica, lupus y tumor pedunculado ${ }^{6}$.

\section{Esclera}

La TBC es una causa rara de escleritis y epiescleritis $^{27}$. El tipo más común es la escleritis focal necrosante y puede ocurrir perforación escleral. La escleritis es una enfermedad ocular inflamatoria poco común, asociada, más frecuentemente, con enfermedades sistémicas inflamatorias de origen autoinmune. La escleritis anterior puede ser causada por infecciones microbianas y la escleritis posterior es, predominantemente, idiopática o autoinmune; cuando su causa es infecciosa, generalmente se debe a extensión de la escleritis anterior. En la revisión realizada sólo se encontró un caso de escleritis posterior en un paciente con TBC sistémica ${ }^{28}$ (Figura 1).

\section{Córnea}

El compromiso corneal se presenta, generalmente, como querato-conjuntivitis flictenular o queratitis intersticial. La querato-conjuntivitis flictenular es considerada una reacción de hipersensibilidad no específica en la córnea o conjuntiva, en respuesta a una proteína extraña. Los pacientes presentan ojo rojo, epífora y sensación de cuerpo extraño. La gravedad de los síntomas corresponde al grado de afectación de la córnea. El epitelio subyacente puede llegar a erosionarse. En áreas donde la $\mathrm{TBC}$ es endémica, ésta es considerada la causa principal ${ }^{29}$. La queratitis intersticial es mayormente unilateral y se caracteriza por ser un infiltrado estromal periférico con vascularización, causado por una reacción alérgica a las proteínas de las micobacteria.

\section{Enfermedad intraocular \\ Úvea}

La prevalencia de uveítis tuberculosa siempre ha sido un tema controversial ${ }^{6}$. A fines del siglo XIX, la TBC era considerada una causa común de uveítis. A mediados del siglo XX, la TBC era considerada como la causa predominante de uveítis granulomatosa. En el año 1952, Guyton y Woods encontraron que $80 \%$ de las uveítis granulomatosas eran causadas por TBC. Sin embargo, estos números declinaron posteriormente. En el año 1960, Woods encontró que sólo $20 \%$ de casos de uveítis eran atribuibles a TBC. Es posible que esta disminución se deba al reconocimiento de otras causas de uveítis granulomatosa, tales como sarcoidosis, toxoplasmosis e histoplasmosis, entre otras. En las últimas décadas, la incidencia ha continuado en descenso, llegando a describirse que la proporción de uveítis tuberculosa en las series varía de 0 a $4 \%{ }^{6}$.

La uveítis tuberculosa era responsable de $10 \%$ de las causas de uveítis en Japón en los años 60. Esta incidencia declinó a $0,3-0,4 \%$ en los años 90 . En el 2002, Morimura encontró en una serie de 179 pacientes referidos por uveítis, que 10 pacientes $(5,6 \%)$, tenían diagnóstico de TBC ocular ${ }^{22}$. La TBC es considerada una causa importante de uveítis en las regiones del mundo en que la prevalencia de la misma es elevada. Por ejemplo, en centros de referencia de enfermedad ocular en la India entre los años 1996 y 2001, 10\% de los casos de uveítis fueron causados por TBC, siendo ésta la causa más común de uveítis ${ }^{25}$. Incidencias similares se han reportado en la literatura médica en Japón y Arabia Saudita ${ }^{22,12}$; en $50 \%$ de los pacientes con uveítis granulomatosa la causa de la lesión fue TBC. El reporte de incidencia de esta enfermedad ha sido reconocido por varios autores en Perú. Por ejemplo, Cerrate reportó en un estudio prospectivo realizado en el Instituto Nacional de Oftalmología, en Perú, la mayor frecuencia de presentación clínica de uveítis posterior con nódulos múltiples coroideos $\mathrm{y}$, menos frecuentemente, uveítis anterior asociada a uveítis y queratitis; ambos cuadros pueden presentarse con o sin enfermedad sistémica ${ }^{30}$. Calderón, en una serie de 113 pacientes con diagnóstico de uveítis, encontró que dentro de las causas más frecuente de uveítis, $11,6 \%$ fueron de origen tuberculoso, siendo ésta la tercera etiología más frecuente de uveítis ${ }^{31}$. 
En la Tabla 3 se resume la tasa de uveítis por tuberculosis en diferentes regiones.

La uveítis anterior es la más frecuente, seguida de la uveítis posterior $^{32}$. La uveítis anterior tuberculosa es granulomatosa en la mayoría de los casos y causa precipitados de queratina, nódulos en el iris, sinequias posteriores y glaucoma secundario. Existen reportes de tuberculosis miliar con siembra en el iris.

En la Tabla 4 se presentan los resultados de otros dos estudios de pacientes con uveítis por TBC (Figura 2).

\section{Coroides}

La TBC se puede presentar como una lesión focal de la coroides, siendo los tubérculos coroideos y los tuberculomas oculares (masas grandes solitarias) las manifestaciones oculares mejor documentadas de la $\mathrm{TBC}^{6,20,33-36}$. Clásicamente, los bacilos tuberculosos causan una respuesta tisular tipo granuloma caseoso, pero puede haber otras causas de reacción granulomatosa, como sífilis, sarcoidosis, toxoplasmosis, lepra, brucelosis, histoplasmosis y toxocariasis ${ }^{37}$. Otras presentaciones menos frecuentes incluyen las hemorragias coroideas diseminadas, las masas coroideas y la coroiditis multifocal ${ }^{33}$. La coroiditis tuberculosa multifocal, aunque poco común, es bien reconocida y puede avalar el diagnóstico de TBC miliar o diseminada como reportó Grewal ${ }^{38}$. Se debe recordar que existen casos de coroiditis solitaria, en los que se presenta

\begin{tabular}{|lcc|}
\hline \multicolumn{2}{|c|}{ Tabla 3. Tasa de uveítis causada por tuberculosis } \\
en Japón, India y Perú
\end{tabular}

un granuloma coroideo solitario, siendo el diagnóstico diferencial TBC, sarcoidosis, toxocariasis, enfermedad por arañazo de gato $\mathrm{u}$ otras enfermedades. No obstante, en muchos casos, la coroiditis solitaria continúa siendo idiopática a pesar de una evaluación sistémica exhaustiva $^{39}$. La TBC coroidal es usualmente asintomática, pero algunos pacientes pueden presentar disminución de la agudeza visual. La infección de la coroides es más frecuente en presencia de enfermedad diseminada.

En la década del cincuenta, se describía que la TBC coroidea podía asumir las siguientes formas ${ }^{16}$ :

Lesiones tuberculosas propiamente dichas:

- TBC miliar aguda: generalmente pasa inadvertida para el paciente pero, cuando los tuberculomas están situados en la mácula o en zonas vecinas, puede ocasionar disminución de la visión. Habitualmente, éstos son más periféricos $\mathrm{y}$, en raras ocasiones, producen escotomas. En cuanto a su evolución, puede haber una regresión simple (no dejar secuela) o una regresión con cicatrización por destrucción corio-retiniana.

- TBC miliar crónica: ocurre, al igual que la anterior, como resultado de la bacilemia. Las alteraciones anatómicas son esencialmente iguales a la anterior pero hay tiempo para una reacción de los tejidos con formación de lesiones granulomatosas progresivas y de lenta evolución. Luego de la formación de un tubérculo miliar agudo, evoluciona hacia la cicatrización, dejando áreas blanquecinas como resultado de la atrofia de las capas coroideas.

- TBC crónica diseminada: es, en realidad, una forma de evolución de la anterior; la atrofia puede ser tan intensa que no quedan zonas sanas. La parte profunda de la esclerótica y la córnea pueden afectase por diseminación periférica produciendo escleroqueratouveítis.

- TBC conglomerada: también se conoce como tubérculo solitario de la coroides. Puede comprometer ambos ojos, aunque frecuentemente se trata de un foco único. Tiene una gran tendencia a progresar y lleva a formas destructivas. Su evolución puede ser

\begin{tabular}{|c|c|c|c|c|c|c|c|c|}
\hline \multirow[b]{2}{*}{$\begin{array}{l}\text { Primer } \\
\text { autor }\end{array}$} & \multicolumn{2}{|c|}{ Uveítis anterior } & \multicolumn{2}{|c|}{ Uveítis intermedia } & \multicolumn{2}{|c|}{ Uveítis posterior } & \multicolumn{2}{|c|}{ Pan-uveítis / "difusa"* } \\
\hline & $\begin{array}{l}n \text { casos } \\
\text { con } D x\end{array}$ & $\begin{array}{l}n \text { casos } \\
\text { con TBC }\end{array}$ & $\begin{array}{l}n \text { casos } \\
\text { con Dx }\end{array}$ & $\begin{array}{l}n \text { casos } \\
\text { con TBC }\end{array}$ & $\begin{array}{l}n \text { casos } \\
\text { con } D x\end{array}$ & $\begin{array}{l}n \text { casos } \\
\text { con TBC }\end{array}$ & $\begin{array}{l}n \text { casos } \\
\text { con Dx }\end{array}$ & $\begin{array}{l}n \text { casos } \\
\text { con TBC }\end{array}$ \\
\hline Singh $(2004)^{25}$ & 235 & $48(7,9 \%)$ & 17 & $8(4 \%)$ & 186 & $22(11,8 \%)$ & 164 & $47(26 \%)$ \\
\hline Islam $(2002)^{12}$ & 119 & $9(7,6 \%)$ & 13 & $2(15,4)$ & 23 & $8(34,8 \%)$ & 45 & $2(4,4 \%)$ \\
\hline
\end{tabular}


regresiva, dejando sólo una cicatriz corio-retiniana, pero también puede avanzar llegando a dar una retinitis proliferante con rotura de la membrana de Bruch. La forma progresiva se comporta como un tumor intraocular, siendo un cuadro de suma gravedad que conduce a la destrucción del globo ocular. Actualmente, son casos raros, debido a que el tratamiento antituberculoso detiene su progresión. La pthisis bulbi es el resultado final en estos casos.

- TBC proliferante difusa: es una forma de evolución rápidamente progresiva y destructiva, con mayor tendencia a la caseificación. Generalmente, terminan en pthisis bulbi. Se trata de la forma más grave de TBC ocular. Se instala de modo brusco, produce grandes signos inflamatorios y destrucción rápida ocasionando ceguera permanente.

\section{Coroiditis granulomatosa tuberculosa}

Es un proceso crónico, con tendencia a la recurrencia y generalmente bilateral. Este proceso recidivante cada vez deja mayor déficit visual y conduce a complicaciones muy graves (glaucoma secundario, etc). En el fondo de ojo se pueden observar dos formas de estas inflamaciones inespecíficas: la coroiditis plástica aguda y la coroiditis exudativa crónica recurrente.

La TBC coroidal puede también presentarse como coroiditis progresiva multifocal o difusa, que puede aparentar ser una coroiditis serpiginosa. Es importante reconocer este tipo de presentaciones porque, en estos casos, hay una buena respuesta al tratamiento antituberculoso sistémico ${ }^{33}$ (Figura 3).

\section{Retina}

El compromiso aislado de la retina es poco frecuente; sin embargo, puede ser el resultado de la invasión directa del bacilo, de la extensión de la enfermedad coroidal o de una respuesta inmunológica al mismo. La aparición de lesiones retinianas en pacientes con TBC sistémica es muy inusual, así se encontró que en sólo $18 \%$ de los pacientes existen lesiones retinianas por $\mathrm{TBC}^{13,26}$.

Las formas tuberculosas retinianas que se describen son las siguientes:

- Miliar: es rara como localización exclusiva pero, generalmente, acompaña a la periflebitis. Los tubérculos, generalmente se originan en la periferia y siguen algún vaso de la retina. Son difíciles de visualizar con la oftalmoscopia. Generalmente, se acompaña de hemorragia retiniana. Si el proceso no se detiene con el tratamiento, puede llegar a la papila y producir ceguera.

- Tubérculo masivo de la retina: es infrecuente como localización única en el ojo. Cuando se presenta, adopta el aspecto tumoral o inflamatorio exudativo.

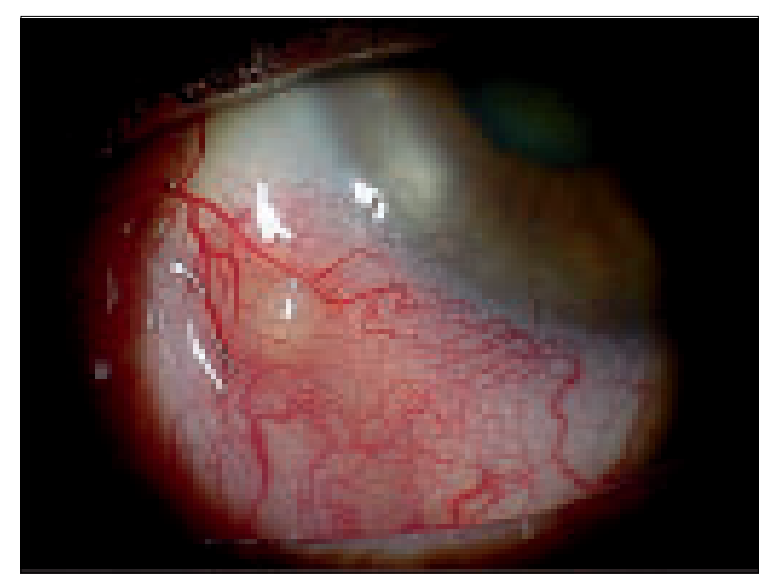

Figura 1. Nódulo escleral en paciente con tuberculosis ocular.

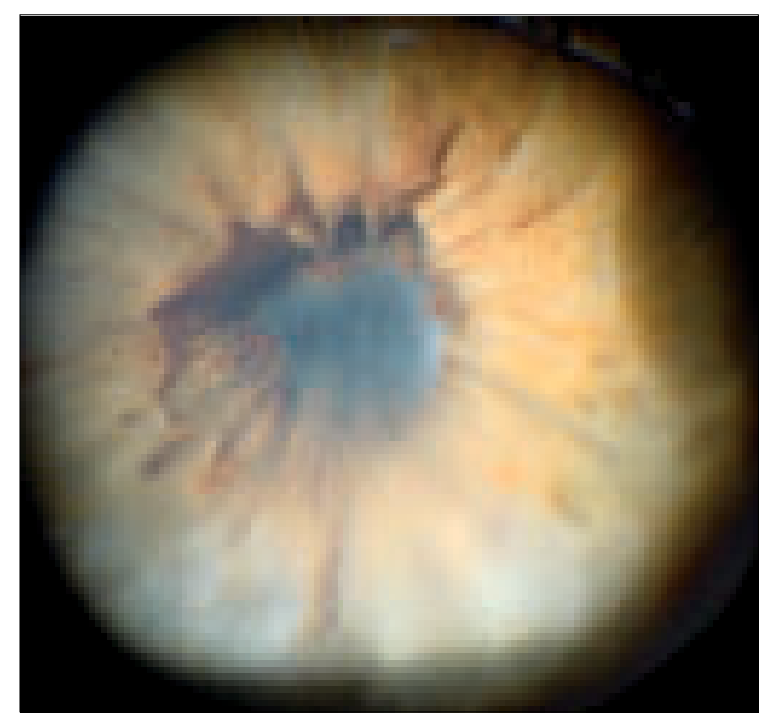

Figura 2. Uveítis anterior con secuela de sinequias anteriores y cataratas.

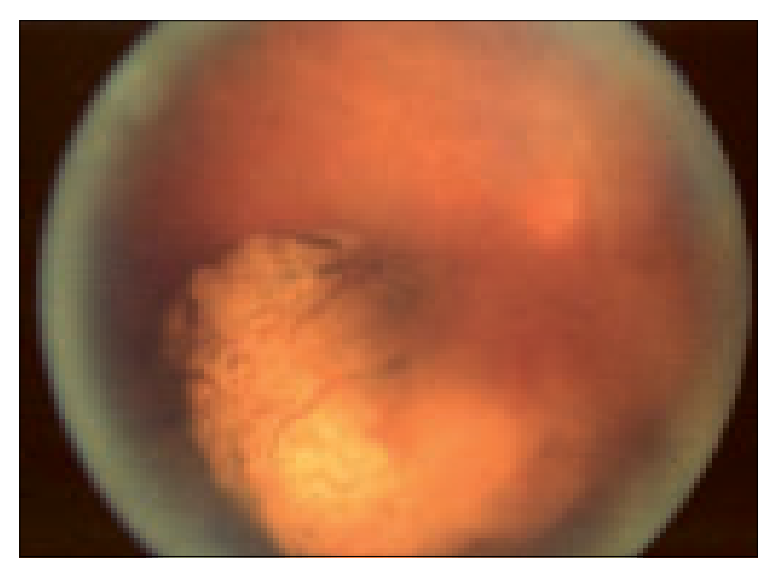

Figura 3. Coroiditis granulomatosa recurrente. 
- Retinitis exudativa tuberculosa: es una forma inusual, debida a una reacción de hipersensibilidad. Puede haber dilatación venosa, hiperemia y, a veces, hemorragia.

- Periflebitis tuberculosa retiniana: o angiopatía retiniana juvenil de Coats. Es la causa más frecuente de hemorragia recidivante del vítreo y enfermedad de Eales. El paciente nota disminución brusca de la visión en un ojo. El diagnóstico diferencial se plantea con diabetes mellitus, hipertensión arterial, arteriosclerosis y desprendimiento de la retina, por ser éstas causas de hemorragia del vítreo.

La enfermedad de Eales fue descrita por primera vez por Henry Eales en 1880. Es una perivasculitis primaria de la retina, que afecta de predominio a la retina periférica ${ }^{40-42}$. Ocurre mayormente en hombres jóvenes (entre los 15 y 40 años), por múltiples causas $^{41}$. El evento inicial en esta enfermedad es una disminución en la visión, súbita, secundaria a una hemorragia del vítreo. Luego que desaparece esta hemorragia aparecen exudados perivasculares y focos hemorrágicos a lo largo de los vasos de la retina. Esta vasculitis puede progresar llegando a trombosis venosa, neo-vascularización, proliferación del tejido glial $\mathrm{y}$, eventualmente, a desprendimiento fraccional de la retina. Mediante RPC se ha detectado ADN de M. tuberculosis en humor vítreo y en la membrana epi-retinal de pacientes con enfermedad de Eales ${ }^{10,41,43}$. Se demuestra así que la TBC es una causa importante de esta enfermedad.

- Periarteritis tuberculosa: es muy rara como proceso aislado. Se produce a nivel de los cruces arteriovenosos y es una manifestación secundaria de la periflebitis.

\section{Pan-oftalmitis}

La pan-oftalmitis es con mayor frecuencia causada por hongos; pocas bacterias han sido reportadas como causantes de esta enfermedad. Según Chawla et al, sólo se han reportado 11 casos de pan-oftalmitis tuberculosa. La mayoría de ellos se ha reportado en individuos inmunocomprometidos, consumidores de sustancias ilegales ${ }^{44}$. Los signos clínicos que sugieren pan-oftalmitis tuberculosa son: ausencia de dolor, presencia de nódulos sobre o dentro del globo ocular y perforación espontánea. Se han reportado casos de pan-oftalmitis y endoftalmitis tuberculosa que se presentaron con síntomas oculares simulando malignidad intraocular o la presencia de un tumor ocular.

\section{Nervio óptico}

En el fondo del ojo se pueden observar dos tipos de lesiones bacilares de la papila: una lesión aislada primitiva (tubérculo solitario) y una lesión metastásica (tu- bérculos miliares). En cuanto al tubérculo solitario, generalmente se observa en niños y jóvenes; por tanto, el diagnóstico diferencial es con retinoblastoma. La mayoría de lesiones se producen en la papila óptica, tomando el aspecto de una masa pseudo-tumoral con edema, hemorragia y enturbiamiento del vítreo. Otras veces puede causar desprendimiento de retina, localizado o total. Los síntomas funcionales son dolor importante y visión nula. En este grupo de alteraciones del nervio de origen metastático, se describen también la trombosis de la vena central secundaria al proceso tuberculoso localizado a nivel de la papila, las lesiones del nervio, secundarias a infecciones tuberculosas procedentes de estructuras vecinas como la retina, tracto uveal, órbita o meninges ${ }^{16}$.

\section{Órbita}

La TBC de la órbita es infrecuente y ocurre por diseminación hematógena o por extensión, a partir de estructuras adyacentes ${ }^{45}$. Es de progresión lenta, crónica y unilateral. Desde el año 1881 hasta el año 1993, se han reportado en la literatura 43 casos de pacientes con evidencia histológica de TBC orbitaria ${ }^{6}$. La mayor cantidad de éstos se concentran en áreas endémicas como la India. Los hallazgos más comunes son: dolor ocular, proptosis, edema palpebral, hinchazón periorbitaria intermitente, cefalea, epistaxis, disminución de la visión, anormalidades en los campos visuales, quemosis, pupila de Marcus Gunn, epífora y aumento de la resistencia orbitaria a la retropulsión ${ }^{10}$. Puede ocurrir también fistulización espontánea de un absceso orbitario. La erosión del hueso es un hallazgo radiológico común ${ }^{6}$.

\section{Métodos diagnósticos}

El diagnóstico de TBC pulmonar se basa en el examen directo de esputo pero, actualmente, un tercio de éstos son negativos. Estas cifras son mayores en países del África, llegando a $80 \%$. En los casos de TBC extra-pulmonar, se utilizan para el diagnóstico otros métodos como el examen directo, cultivo, anatomía patológica $\mathrm{y}$, recientemente, métodos más modernos como la RPC, entre otros ${ }^{46}$.

El diagnóstico de TBC ocular generalmente es presuntivo, se basa en la apariencia clínica, evaluación sistémica y respuesta al tratamiento ${ }^{48,49}$. El diagnóstico definitivo se hace con la detección de M. tuberculosis en fluidos o tejidos ${ }^{11,49}$. Debido a la buena respuesta terapéutica, un diagnóstico temprano es lo más deseado. La terapia antituberculosa es prolongada y tóxica y en muchos casos se administra de manera empírica, por lo que se busca un diagnóstico confirmado. 
Cuando se presenta en la consulta un paciente con infección sistémica, ojo rojo doloroso o disminución de la visión, los médicos no oftalmólogos no tienen la habilidad o los instrumentos disponibles para hacer un diagnóstico preciso, y en la mayoría de los casos, el diagnóstico es presuntivo.

A pesar del uso de herramientas moleculares altamente sensibles para el diagnóstico de infección por M. tuberculosis, la TBC ocular aún continúa siendo una patología de difícil reconocimiento ${ }^{5,50}$. El diagnóstico debe comenzar con un examen físico completo, examen de esputo (frotis y cultivo), prueba del derivado de la proteína purificada (PPD) y una radiografía de tórax ${ }^{10}$.

Para el diagnóstico de TBC ocular se usan las siguientes categorías:

- Diagnóstico definitivo: lesión pulmonar o extrapulmonar, con baciloscopia (BK) $(+)$ en esputo, líquido o en anatomía patológica, asociado a lesión ocular. Asimismo, se debe considerar como diagnóstico definitivo una RPC positiva en humor acuoso o vítreo o (con) $\mathrm{BK}(+)$ de humor acuoso o vítreo.

- Diagnóstico probable: lesiones pulmonares y/o extra-pulmonares, con BK (-) y PPD (+).

- Diagnóstico posible: lesión ocular sin lesión pulmonar, con PPD positivo.

En la Tabla 5 se resumen los resultados de pacientes con uveítis y su diagnóstico, en tres series clínicas.

Si las pruebas iniciales son negativas, esto no debe excluir a la TBC en el diagnóstico diferencial. Abrams y Schlaegel $^{51}$, reportaron en el año 1982, 18 pacientes con TBC presuntiva; de éstos, 17 casos tenían una radiografía sin evidencia de enfermedad activa y sólo nueve tuvieron una prueba de PPD positiva. Estos 18 pacientes fueron diagnosticados como uveítis tuberculosa y recibieron la prueba terapéutica de isoniacida (HIN) con buena respuesta terapéutica.
Prueba terapéutica con isoniacida: consiste en la administración de $300 \mathrm{mg}$ de isoniacida al día, durante tres semanas. Se considera un resultado positivo si hay una mejora significativa en las semanas 1 a 3 de tratamiento, debiendo extenderse la terapia hasta completar los seis meses ${ }^{6}$. Esta práctica actual no es promovida ni aceptada por los conceptos básicos de tratamiento de la TBC.

Frotis y cultivo. El diagnóstico definitivo de la TBC requiere el aislamiento de $M$. tuberculosis desde los tejidos o fluidos oculares. La preparación de la muestra de humor acuoso o vítreo para la tinción ácido alcohol resistente (Ziehl Neelsen), requiere concentrar los fluidos por el método de centrifugación citológica; aún así, la cantidad de micobacterias presentes es extremadamente pequeña, reduciéndose la sensibilidad diagnóstica del estudio ${ }^{8}$.

La obtención de muestras para cultivos puede ser simple, si estamos frente al caso de enfermedad externa pero, en la mayoría de casos de TBC ocular, la obtención de muestra de los tejidos involucrados es poco práctica ${ }^{53}$. Cuando se ha realizado una punción para extraer humor acuoso o vítreo, la positividad del cultivo ha sido baja ${ }^{10}$.

Lowenstein Jensen es el medio utilizado para el aislamiento de M. tuberculosis. Los cultivos son incubados por lo menos ocho semanas y se detectan colonias visibles a partir del primer mes. Hasta este momento, el aislamiento de M. tuberculosis en el cultivo continúa siendo el estándar de oro para el diagnóstico final de TBC. La incorporación de métodos de cultivo líquidos rápidos, usados en conjunto con los medio sólidos en los protocolos de laboratorio, ha permitido acelerar el crecimiento de M. tuberculosis ${ }^{53}$.

$P P D$. Es el método estándar para hacer el diagnóstico de infección por $M$. tuberculosis. Es un método simple y barato; muy útil para dar profilaxis en quienes han tenido un viraje tuberculínico reciente y se reco-

\begin{tabular}{|c|c|c|c|c|c|c|c|}
\hline & $\begin{array}{l}\text { Pacientes con } \\
\text { uveítis EAD }\end{array}$ & Evaluación clínica $(+)^{*}$ & & D (+) & $\begin{array}{l}\text { TBC intraocular } \\
\text { (Dx probable) }\end{array}$ & $\begin{array}{l}\text { TBC ir } \\
\text { (Dx d }\end{array}$ & $\begin{array}{l}\text { ntraocular } \\
\text { lefinitivo) }\end{array}$ \\
\hline Morimura et al (2002) (ref 22) & 179 & 126 & & $(14,5 \%)$ & $10 \quad(5,6 \%)$ & & - \\
\hline Islam et al (2002) (ref 12) & 200 & ND & & ND & $21(10,5 \%) * *$ & & - \\
\hline Singh et al (2004) (ref 25) & 1.233 & ND & & ND & - & 125 & $(10,1 \%)$ \\
\hline \multicolumn{8}{|c|}{$\begin{array}{l}\text { *Presencia de una o más de las siguientes características: uveítis recurrente; uveítis grave que compromete el segmento posterior; síntomas sistémicos asociados; } \\
\text { sospecha de enfermedad sistémica como causa de uveítis; intención de tratar al paciente con medicación sistémica. A estos pacientes se les realizó una evaluación } \\
\text { sistémica que incluyó una prueba de PPD. } \\
\text { **Diagnóstico clínico sobre la base de la presencia de uveítis anterior granulomatosa y no granulomatosa, con grados variables de sinequia posterior, corio- } \\
\text { retinitis geográfica o multifocal, un PPD }(+) \text { y respuesta a terapia antituberculosa sin corticoesteroides sistémicos. } \\
\text { ND = no determinado. } \\
\text { EAD = etiología a determinar }\end{array}$} \\
\hline
\end{tabular}


noce como prueba positiva cuando es $\geq 10 \mathrm{~mm}$. Debe recordarse que en pacientes inmunocomprometidos, como infección por VIH /SIDA u otros, el punto de corte es de $5 \mathrm{~mm}$. En áreas endémicas, la tasa de PPD es creciente según la edad y en Perú a los 20 años; 40 a $50 \%$ de la población sana tiene la infección, llegando a afectar el $50 \%$ de la población adulta sana. La dificultad de la interpretación en pacientes con BCG al nacer y un refuerzo al inicio de la edad escolar, hace que la especificidad de una prueba positiva sea mucho menor, perdiendo su valor diagnóstico y su utilidad clínica. En países con alta prevalencia de TBC, la vacunación con BCG es rutinaria. Esto conlleva a que estas personas tengan un PPD positivo sin tener la enfermedad y se deben realizar pruebas adicionales para confirmar el diagnóstico de TBC. Por el contrario, en zonas donde la TBC no es endémica, un PPD positivo tendría un mayor valor diagnóstico.

Recientemente, la Food and Drug Administration (FDA) aprobó el Quantiferon ${ }^{\circledR}$ prueba que mide la producción de interferón gama por los linfocitos del paciente expuestos a antígenos tuberculosos purificados; examen que permite diferenciar la respuesta inmune de $M$. bovis contenido en la vacuna BCG de la infección natural por $M$. tuberculosis.

Se ha reportado que entre 2 y $56 \%$ de los pacientes con formas extra-pulmonares de TBC tienen un resultado de PPD negativo, dependiendo del lugar donde se encuentre la infección. Incluso, se han reportado casos de pacientes con TBC coroidal comprobada por biopsia, en los cuales se había descrito un PPD negativo previamente. Por tanto, es posible que en los estudios en los cuales se toma como punto de corte un PPD positivo (mayor de $10 \mathrm{~mm}$ de induración), como criterio diagnóstico para empezar un plan de trabajo de búsqueda activa de TBC como causa de la lesión ocular, se estén perdiendo algunos pacientes en los cuales el diagnóstico pudiera también ser TBC. En conclusión, el realizar una prueba de PPD es considerada de valor diagnóstico en TBC ocular, aunque pueden darse casos de falsos negativos ${ }^{7}$.

Reacción de polimerasa en cadena. La amplificación del ADN de M. tuberculosis por la RPC se está usando con más frecuencia para el diagnóstico de TBC ocular y de otros órganos ${ }^{55}$. En los últimos años, la RPC se ha usado en el diagnóstico de TBC ocular y ha abierto una nueva opción para el diagnóstico microbiológico, ofreciendo ventajas sobre los métodos tradicionales para la identificación de $M$. tuberculosis. La RPC puede detectar ARN o ADN de cualquier microorganismo, con una sensibilidad y especificidad mayor que la del cultivo. Por ello se está convirtiendo en el método diagnóstico de elección. Además, es una prueba de rápido resultado y que requiere una peque- ña cantidad de muestra, la que es obtenida por punción ocular. El análisis de la muestra obtenida por punción ocular permite diferenciar entre una etiología infecciosa y no infecciosa, en especial, cuando se desea descartar malignidad. Dentro de las complicaciones reportadas al realizar este procedimiento, se describe el desprendimiento de retina ${ }^{56}$. En áreas donde la TBC es endémica y las reacciones positivas a la tuberculina son comunes, como en el caso de Perú, la RPC ayudaría a detectar pacientes que podrían obtener un gran beneficio del tratamiento anti-tuberculoso al proveer una evidencia objetiva de infección por $M$. tuberculosis en pacientes con inflamación ocular ${ }^{19}$.

Kotake et al, reportaron por primera vez el uso de RPC en el diagnóstico de TBC ocular. Usaron muestras de humor acuoso de dos pacientes con vasculitis retinal activa. Los pacientes respondieron bien al tratamiento anti-tuberculoso. En 1999, Arora y cols, evaluaron la eficacia diagnóstica de esta técnica en pacientes con uveítis granulomatosa activa ${ }^{57}$. Obtuvieron muestras de humor acuoso de 53 pacientes con una reacción celular en la cámara anterior, junto con uno o más de los siguientes hallazgos: vasculitis activa, células de humor vítreo de la cámara anterior, opacidades "en copo de nieve", depósitos "en copo de nieve" en la pars clara y retino- coroiditis. Las muestras fueron sometidas a RPC. Se usaron muestras de 17 pacientes con un diagnóstico diferente a TBC para formar el grupo control. Además, se obtuvo muestras de 15 pacientes sanos que fueron sometidos a cirugía de catarata de rutina, para usarlos como control del paciente sano. De las 53 muestras, veinte $(37,7 \%)$ fueron positivas para TBC, sólo una en el grupo de control de enfermedad fue levemente positiva y ninguna en el grupo control del paciente sano. Este estudio demostró que la RPC puede ser usada, efectivamente, en el diagnóstico de TBC ocular en la presencia de uveítis.

Biswas y cols, en un estudio realizado en el año 1999, utilizaron la técnica de RPC con el partidor IS6110 para detectar la presencia de ADN de M. tuberculosis en el humor vítreo de un paciente con enfermedad de Eales y en un grupo control, encontrando una diferencia estadísticamente significativa $(p=0,01)$ de TBC en esta enfermedad. V. Gupta y cols, han reportado una sensibilidad de $37 \%$ para la prueba de RPC en el diagnóstico de TBC ocular ${ }^{19}$. En otro artículo publicado por los mismo autores, se encontró que la sensibilidad de la prueba de RPC fue de $72 \%{ }^{3}$. De acuerdo con estos autores, el incremento en la sensibilidad de esta prueba se debe a una mejor aplicación de criterios para la selección de la población de pacientes estudiados. Adicionalmente en este trabajo, a diferencia del anterior, se realizó punción de humor vítreo además de la 
punción de humor acuoso. Creemos que esto podría haber contribuido al aumento en la sensibilidad.

Reacción de polimerasa en cadena anidada. La técnica de RPC anidada (nested PCR en inglés), específica para el gen MPB64, descrita por Narita y cols ${ }^{56}$, fue estandarizada por Madhavan y cols ${ }^{43}$. MPB64 es una proteína altamente inmunogénica que se encuentra en los cultivos filtrados no calentados de $M$. tuberculosis y algunas cepas de $M$. bovis $\mathrm{BCG}^{59}$. Se encontró que la técnica de RPC anidada fue no sólo específica, sino también 10 mil veces más sensible que la RPC usando partidores de IS6110. La secuencia de inserción IS6110 es relativamente estable a lo largo de meses, pero tiene una tasa de transposición observable, conforme pasa el tiempo ${ }^{60}$. Estudios recientes realizados en Chennai, en el Sur de India, han demostrado que $40 \%$ de los aislados locales de M. tuberculosis tienen sólo una copia o ninguna de IS6110 ${ }^{61}$. Más aún, Doucet-Populaire y cols, encontraron también que la técnica de RPC, usando el IS6110, es poco sensible. Teniendo en consideración estos hallazgos, y con el objetivo de buscar un partidor más sensible, surge el enfoque de RPC anidada con codificación genética para MPB64. Se encontró que esta técnica era $100 \%$ más específica y 10 mil veces más sensible en detectar la presencia de hasta un solo bacilo tuberculoso en una muestra ${ }^{61}$.

\section{Tratamiento}

Para tratar una infección, el antimicrobiano debe ser capaz de alcanzar el tejido deseado. Sin embargo, la penetración del fármaco puede estar restringida por las barreras anatómicas que limitan la biodisponibilidad de los antimicrobianos tópicos y sistémicos. El acceso al tejido ocular infectado por los agentes sistémicos está limitado por la falta de vascularización ocular, particularmente en la cornea y el vítreo ${ }^{23}$.

El esquema de primera línea es el uso de cuatro fármacos anti-tuberculosos, diarios, durante dos meses y luego, dos fármacos por cuatro meses. Los cuatro anti-tuberculosos de primera línea son isoniacida, rifampicina (RFP), pirazinamida (PZD) y etambutol; se dan en dosis única diaria. En la segunda fase se ha recomendado dos a tres veces por semana HIN + RFP. Recientemente ha ido en incremento la tasa de TBC multiresistente-MDR, resistente a HIN/RFP, que requiere tratamiento por 24 meses, con cinco a seis antituberculosos, en terapia diaria.

Morimura y cols, en Japón, refieren que el tratamiento consiste en HIN $400 \mathrm{mg}$ por día, con la posibilidad de añadir RFP $450 \mathrm{mg}$ por día, mayormente en los casos que están asociados a infección por VIH, ya que la tasa de resistencia al tratamiento anti-tuberculoso, es baja en Japón. Por tanto, en este estudio, sólo tres de los siete pacientes, recibieron terapia combinada ${ }^{22}$. El tratamiento debe ser durante seis meses, idealmente, aunque en algunos casos no se llega a completar este tiempo de tratamiento por la falta de adherencia al mismo. Además, en algunos casos se indica el uso de corticosteroides orales como prednisolona a una dosis de 30 a $40 \mathrm{mg}$ por día. Según Morimura, los corticosteroides orales están indicados en pacientes en los que la inflamación afecta el disco óptico o la mácula, o por la presencia de neo-vascularización retinal de etiología inflamatoria ${ }^{22}$. El uso de corticosteroides locales u orales es frecuente, por dos a cuatro semanas, en los casos de uveítis o epi-escleritis. Se estima que en presencia de lesión ocular, el tratamiento debe ser igual que en los otros casos de TBC extrapulmonar, con un tiempo de tratamiento de seis meses. En nuestra experiencia, el uso de terapia bi-semanal en la segunda fase, se ha asociado a algunas recaídas y, por la misma razón, recomendamos terapia diaria en los casos de uveítis o vitritis grave, también en esta fase.

El tratamiento debe ser bajo la política de DOTS (directamente observada) que recomienda la OMS. Algunos autores como Morimura recomiendan tratamiento de pruebas con HIN, con o sin RFP, evalúan al cabo de tres semanas y toman decisiones de completar seis meses con tratamiento completo si hubiera mejoría $^{22}$. No compartimos esta recomendación. El uso de corticosteroides, está indicado en uveítis grave, especialmente en pan-uveítis, por el pobre pronóstico. Esta terapia debe ser oral y sólo por dos a tres semanas. Algunos autores lo recomiendan en lesiones del nervio óptico o neo-vascularización de la retina. También se ha usado corticosteroides tópico en uveítis anterior, con buen pronóstico. Woods y cols, describieron que el uso de corticosteroides sistémicos, en combinación con tratamiento anti-tuberculoso, se asocia a un curso prolongado de TBC y una alta incidencia de recurrencia. De otra parte, el uso de corticoesteroides tópicos, en combinación con cicloplejia, puede ser útil en el tratamiento de reacciones de la cámara anterior y la prevención de la formación de sinequias $^{2}$. En un estudio en la India, en el cual se usó la RPC positiva como diagnóstico de $\mathrm{TBC}$, los pacientes recibieron terapia anti-tuberculosa y corticosteroides sistémicos o locales. El esquema fue: HIN/RFP/PZD durante 2 a 3 meses y en la segunda fase HIN/RFP por otros 9 a 12 meses, obteniendo resultados satisfactorios.

\section{Resumen}

La tuberculosis (TBC) es considerada un problema de salud pública en varios países del mundo. Esta 
enfermedad se clasifica en pulmonar y extra pulmonar. Dentro de la forma extra pulmonar, el compromiso ocular es poco común, pero es importante conocerlo ya que su incidencia puede llegar hasta $1 \%$. Los casos de $\mathrm{TBC}$ ocular se dividen en primarios y secundarios. Las manifestaciones oculares pueden ser causadas por una infección activa que invade el ojo o por una reacción inmunológica de hipersensibilidad retardada, en ausencia del agente infeccioso. Las presentaciones más comunes son: uveítis anterior crónica, coroiditis y esclero-queratitis. A pesar de la existencia de herramientas moleculares altamente sensibles, el diagnóstico de TBC ocular continúa siendo presuntivo, basándose en la presentación clínica, evaluación sistémica y la respuesta terapéutica. Para el tratamiento se utilizan cuatro fármacos por dos meses (isoniacida, rifampicina, pirazinamida y etambutol) y dos fármacos por cuatro meses adicionales.

\section{Referencias}

1.- Pan American Health Organization. Health situation in the Americas, Basic Indicators. 2005. www.paho.org. (accedido en septiembre 2006).

2.- Demirci H, Shields C L, Shields J A, Eagle R C Jr. Ocular tuberculosis masquerading as ocular tumors. Surv Ophthalomol 2004; 49: 78-89.

3.- Sahu G, Mishra N, Bhuita R, Mohanty A. Manifestations in ocular tuberculosis. Ind $\mathrm{J}$ Tub 1998; 45: 153-4.

4.- WHO TB country profile Peru. WHO Report 2006 Global Tuberculosis Control: Surveillance, Planning, Financing. www.who.org. (accedido en septiembre 2006).

5.- Bodaghi B, LeHoang P. Ocular tuberculosis Curr Opin Ophthalmol 2000; 11: 443-8. Review.

6.- Helm C J, Holland G N. Ocular tuberculosis. Surv Ophthalmol 1993; 38: 229-56. Review.

7.- Bowyer J D, Gormley P D, Seth R, Downes R N, Lowe J. Choroidal tuberculosis diagnosed by polymerase chain reaction. A clinicopathologic case report. Ophthalmology 1999; 106: 290-4.

8.- Martínez M, Márquez K, Stangogiannis E, Romero B, Padilla R. Uveítis por tuberculosis en el Hospital Universitario de Caracas durante el periodo 2003 -2004. RFM 2004; 27: $157-62$.

9.- Torres R M, Calonge M. Macular edema as the only ocular finding of tuberculosis. Am J Ophthalmol 2004; 138: 1048-9.

10.- Abrams J, Schlaegel T F Jr. The role of isoniazid therapeutic test in tuberculous uveitis. Am J Ophthalmol 1982; 94: 511-5

11.- Levecq LJ, De Potter P. Solitary choroidal tuberculoma in an immunocompetent patient. Arch Ophthalmol 2005; 123: 8646.

12.- Islam S M, Tabbara K F. Causes of uveitis at The Eye Center in Saudi Arabia: A retrospective review. Ophthalmol Epidemiol 2002; 9: 239-49.

13.- Romero P, Castro A, Martínez I, Almena M,
Salvat M, Zamora J. Retinitis por tuberculosis asociada a periflebitis. Arch Soc Esp Oftalmol 2004; 79: 81-4.

14.- Okada A A, Forrester J V. Ocular inflammatory disease in the New Millennium. Arch Ophthalmol 2000; 118 : 116-9.

15.- Varma D, Anand S, Reddy A R, Das A, Watson J P, Currie DC, et al. Tuberculosis: an under-diagnosed aetiological agent in uveitis with an effective treatment. Eye 2006; 20: 1068-73.

16.- Rodríguez R, Massera M J. Manifestaciones oftalmoscópicas de la tuberculosis ocular, Rodriguez R, Massera MJ, Fondo de Ojo, Buenos Aires: Editorial Intermédica; 1959, p. 216-33.

17.- Sarvananthan N, Wiselka M, Bibby K. Intraocular tuberculosis without detectable systemic infection. Arch Ophthalmol 1998; 116: 1386-8.

18.- Sheu S J, Shyu J S, Chen L M, Chen Y Y, Chim S C, Wang J S. Ocular manifestations of tuberculosis. Ophthalmology 2001; 108: 1580-5.

19.- Gupta V, Arora S, Gupta A, Ram J, Bambery P, Sehgal S. Management of presumed intraocular tuberculosis: possible role of the polymerase chain reaction. Acta Ophthalmol Scand 1998; 76: 679-82.

20.- Gupta V, Gupta A, Sachdeva N, Arora S, Bambery P. Successful management of tubercular subretinal granulomas. Ocul Immunol Inflamm 2006; 14: 35-40.

21.- Donahue H C. Ophthalmologic experience in a tuberculosis sanatorium. Am J Ophthalmol 1967; 64: 742-8.

22.- Morimura Y, Okada A, Kawahara S, Miyamoto Y, Kawai S, Hirakata A, et al. Tuberculin skin testing in uvetis patients and treatment of presumed intraocular tuberculosis in Japan. Ophthalmology 2002; 109: 851-7.

23.- Shanmugam M P, Nandakumar M. Ocular tuberculosis masquerading as ocular tumors. Surv Ophthalmol 2004; 49: 619-20.

24.- Raina U K, Tuli D, Arora R, Mehta D K,
Taneja M. Tubercular endophthalmitis simulating retinoblastoma. Am J Ophthalmol 2000; 130: 843-5.

25.- Singh R, Gupta V, Gupta A. Pattern of uveitis in a referral eye clinic in North India. Indian J Ophthalmol. 2004; 52: 121-5

26.- Bouza E, Merino P, Muñoz P, SánchezCarrillo C, Yáñez J, Cortés, C. Ocular tuberculosis: A prospective study in a general hospital. Medicine (Baltimore) 1997; 76: 53-61.

27.- Nanda M, Pflugfelder S, Holland S. Mycobacterium tuberculosis scleritis. Am J Ophthalmol 1989; 108: 736-7.

28.- Gupta A, Gupta V, Pandav S S, Gupta A. Posterior scleritis associated with systemic tuberculosis. Indian J Ophthalmol 2003; 51: 347-9.

29.- Rohatgi J, Dhaliwal U. Phlyctenular eye disease: a reappraisal. Jpn J Ophthalmol 2000; 44: 146-50.

30.- Cerrate A. Adenosina deaminasa en humor acuoso para el diagnóstico de tuberculosis intraocular [Tesis de Magíster en Medicina]. Lima (Pe): UPCH; 2006.

31.- Calderón P. Uveítis: aspectos clínicos, terapéuticos y preventivos [Tesis de bachiller en Medicina]. Lima (Pe): UPCH; 1987.

32.- Rodríguez A, Calonge M, Pedroza-Seres M, Akova Y A, Messmer E M, D'Amico D J, et al. Referral patterns of uveitis in a tertiary eye care center. Arch Ophthalmol 1996; 114: 593-9.

33.- Gupta V, Gupta A, Arora S, Bambery P, Dogra M R, Agarwal A. Presumed tubercular serpiginouslike choroiditis. Clinical presentations and management. Ophthalmology 2003; 110: 1744-9.

34.- Grosse V, Bange F C, Tischendorf J, Schmidt R E, Manns M P. A mass in the eye. Lancet 2003; 360: 922.

35.- Salman A, Parmar P, Rajamohan M, Thomas P A, Jesudasan N. Subretinal fluid analysis in the diagnosis of choroidal tuberculosis. Retina 2003; 23: 796-9.

36.- Mehta S. Ocular lesions in acute 
disseminated tuberculosis. Ocul Immunol Inflamm 2004; 12: 311-5.

37.- Sharma P M, Singh R P, Kumar A, Prakash G, Mathur M B, Malik P. Choroidal tuberculoma in miliary tuberculosis. Retina 2003; 23: 101-4.

38.- Grewal A, Kim R Y, Cunningham E T Jr. Miliary tuberculosis. Arch Ophthalmol 1998; 116: 953-4.

39.- Shields J, Shields C, Demirci H, Hanovar S. Solitary idiopathic choroiditis. Arch Ophthalmol 2002; 120: 311-9.

40.- Moldow B, La Cour M. Eales' disease in Inuit: Report of four cases. Acta Ophthalmol Scand 2003; 81: 304-8.

41.- Biswas J, Therese L, Madhavan H N. Use of polymerase chain reaction in detection of Myocbacterium tuberculosis DNA from vitreous samples of Eales' disease. Br J Ophthalmol 1999; 83: 994.

42.- Shah S M, Howard R S, Sarkies N J, Graham E M. Tuberculosis presenting as retinal vasculitis. J R Soc Med 1988; 61: 232-3.

43. - Madhavan H N, Therese K L, Gunisha P, Jayanthi U, Biswas J. Polymerase chain reaction for detection of Mycobacterium tuberculosis in epiretinal membrane in Eales' disease. Invest Ophthalmol Vis Sci 2000; 41: 822-5.

44.- Chawla R, Garg S, Venkatesh P, Kashyap S, Tewari H K. Case report of tuberculosis panophthalmitis. Med Sci Monit 2004; 10: CS57-59.

45.- Shome D, Honavar S G, Vemuganti G K, Joseph J. Orbital tuberculosis manifesting with endophthalmos and causing a diagnostic dilemma. Ophthal Plast Reconstr Surg 2006; 22: 219-21.

46.- Cunningham E, Rathinam S. TB or not TB? The perennial question. $\mathrm{Br} \mathrm{J}$ Ophthalmol 2001; 85: 127-8.

47. - Sakai J, Matsuzawa S, Usui M, Yano I. New diagnostic approach for ocular tuberculosis by ELISA using the cord factor as antigen. Br J Ophthalmol 2001; 85: 130-3.

48.- Sharma P M, Singh R P, Kumar A, Prakash G, Mathur M B, Malik P. Choroidal tuberculoma in miliary tuberculosis. Retina 2003; 23: 101-4.

49. - Joshi J M, Vijayan V K, Jindal S K, Jagganath K, Rodrigues C, Gupta S B. API TB Consensus Guidelines 2006: Management of pulmonary tuberculosis, extra-pulmonary tuberculosis and tuberculosis in special situations. JAPI 2006; 54: 219-34.

50.- Nahid P, Pai M, Hopewell P C. Advances in the diagnosis and treatment of tuberculosis. Proc Am Thorac Soc 2006; 3: 103-110.

51.- Bonfioli A A, de Miranda S S, Campos W R, Orefice F. Tuberculosis. Semin Ophthalmol 2005; 20: 169-75.

52.- Kuruvilla A. Ocular tuberculosis. Lancet 2003; 361: 260-1.

53.- Heifets L. Dilemas and realities of rapid diagnostic tests for tuberculosis. Chest 2000; 118: $4-5$

54.- Gupta V, Gupta A, Arora S, Sachdeva N, Bambery P. Simultaneous choroidal tuberculoma and epididymo-orchitis caused by Mycobacterium tuberculosis. Am J Ophthalmol 2005; 149: 310-2.

55.- Lobo A, Lightman S. Vitreous aspiration needle tap in the diagnosis of intraocular inflammation. Ophthalmology 2003; 110 595-9.

56.- Narita M, Matsuzono Y, Shibata M, Togashi T. Nested amplification protocol for the detection of Mycobaterium tuberculosis. Act Paediatr 1992; 81: 997-1001.

57.- Arora S K, Gupta V, Gupta A, Bambery P, Kapoor G S, Sehgal S. Diagnostic efficacy of polymerase chain reaction in granulomatous uveitis. Tubercle Lung Dis. 1999; 79: 22933.

58.- Therese K L, Jayanthi U, Madhavan H N. Application of nested polymerase chain reaction (nPCR) using MBP 64 gene primers to detect Mycobacterium tuberculosis DNA in clinical specimens from extrapulmonary tuberculosis patients. Indian J Med Res 2005; 122: $165-70$

59. - Tanaka M M, Small P M, Salamon H, Feldman M W. The dynamics of repeated elements: Applications to the epidemiology of tuberculosis. Proc Natl Acad Sci USA 2000; 97: 3532-7.

60.- Das S, Paramasivan C N, Lowrie D B, Prabhakar R, Narayanan P R. IS 6110 restriction fragment length polymorphism typing of clinical isolates of Mycobacterium tuberculosis from patients with pulmonary tuberculosis in Madras, South India. Tubercle Lung Dis 1995; 76: 550-4.

61.- Doucet-Populaire F, Lalande V, Carpentier E, Bourgoin A, Dailloux M, Bollet $\mathrm{C}$, et al. A blind study of the polymerase chaín reaction for the detection of Mycobacterium tuberculosis DNA. Azay Mycobacteria Study Group. Tubercle Lung Dis 1996; 77: 358-62. 